\title{
Biología, Daño y Control Químico del Gorgojo de los Andes, Rhigopsidius tucumanus Heller
}

\author{
Oscar Barea M., ${ }^{1}$ René Andrew, ${ }^{2}$ Jorge Vargas $\mathrm{O}^{3}$
}

\begin{abstract}
Resumen
El gorgojo de los Andes presente en la zona de Lequezana se identificó como Rhigopsidius tucumanus. Esta plaga desarrolló su ciclo vital de manera sincronizada con el crecimiento del cultivo y duró de 206-290 días con sólo una generación al año. El seguimiento efectuado en el almacén confirmó el desarrollo y crecimiento del insecto dentro el tubérculo. El daño que ocasionó en el campo, en ausencia de control, varió entre 28.3 y 85\%. Las técnicas de mayor efectividad de control fueron: la aplicación de carbofuran granulado (Curater 5 G) a la siembra; a la siembra y aporque en una dosis de $25 \mathrm{~kg} / \mathrm{ha}$; y líquido (Carbodan 48 FW) a la siembra, emergencia, y aporque, con una dosis de 5 //ha.
\end{abstract}

Palabras claves adicionales: $\quad$ plaga, gorgojo, Rhigopsidius tucumanus, carbofuran.

\section{Biology, Damage, and Chemical Control of the Andean Potato Weevil, Rhigopsidius tucumanus Heller}

\section{Summary}

The Andean weevil present in the area of Lequezana was identified as Rhigopsidius tucumanus. The life cycle of this pest proved to be synchronized with the growth of the crop and

Aceptado para publicación: mayo 1997.

1. Investigador IBTA-PROINPA, Programa de Investigación de la PapaRegional Chuquisaca Convenio: IBTA-CIP-COSUDE, Casilla Postal 405, Sucre-Bolivia.

2. Investigador IBTA-PROINPA, Oficina Central, Casilla Postal 4285 Cochabamba, Bolivia.

3. Tesista IBTA-PROINPA, Regional Potosí. 
lasted from 206 to 290 days, with only one generation per year. Monitoring during the storage period confirmed the development and growth of the insect inside the tuber. The damage caused in fields without control measures varied between 28.3 and $85 \%$. Control practices that showed the highest efficiency were: the application of granulated carbofuran (Curater $5 \mathrm{G}$ ) at planting time; at planting and hilling-up time at a rate of $25 \mathrm{~kg} / \mathrm{ha}$; and in liquid form (Carbodan $48 \mathrm{FW}$ ) at planting time, emergence, and hilling-up at a rate of $5 \mathrm{l} / \mathrm{ha}$.

\section{Additional Index words: plague, weevil, Rhigopsidius tucumanus, carbofuran.}

El gorgojo de los Andes por su amplia distribución y por las pérdidas que ocasiona en el campo y almacén, es la plaga de mayor importancia en las zonas paperas del departamento de Potosí (2). El desconocimiento del nivel de daño producido por las larvas del gorgojo y su efecto en el rendimiento, sugiere la necesidad de estudiar la biología y el daño que produce el insecto al tubérculo durante el desarrollo del cultivo y en condiciones de almacenamiento.

Una práctica de combate común entre los agricultores es la utilización de diferentes productos químicos, en diversos modos y épocas de aplicación. Es posible que la falta de efectividad en el uso se origine por el escaso conocimiento de la biología del insecto, su relación con el cultivo y el manejo de plagas y los plaguicidas.

Por lo indicado, los objetivos de esta investigación se dirigen tanto a la identificación de la especie y al estudio de su biología, así como a evaluar y cuantificar el daño que ocasionan el insecto en el campo y almacén, para finalmente determinar el control más adecuado del insecto, a través de épocas y formas de aplicación de insecticidas y el uso de barreras naturales.

\section{Materiales y Métodos}

La biología del insecto se estudió bajo condiciones de laboratorio, en la E.E. Chinoli Potosí. Se inició con la recolección de adultos, y luego del sexado se aislaron por parejas en recipientes de plástico. Iniciada la oviposición, los huevos se colocaron en placas petri con papel secante para su incubación. 
Después de eclosionar las larvas, éstas fueron depositadas en tubérculos partidos, permaneciendo las larvas en los mismos hasta completar su desarrollo.

La evaluación del daño del insecto en campo se realizó en tres localidades en parcelas con y sin aplicación de insecticidas, con la variedad Sani Imilla. El daño al follaje se evaluó en los surcos laterales, cada 14 días y para determinar el daño al tubérculo, se utilizó una escala de 0 a 4 . Para el seguimiento en almacén, se evaluaron 600 tubérculos distribuidos al azar en 10 bolsas red por tratamiento y se observaron cada 14 días hasta noviembre, época de la próxima siembra.

Para el manejo del insecto en campo se evaluaron diferentes épocas de aplicación de insecticidas y una barrera vegetal en dos localidades, los tratamientos fueron: Carbofuran (5G) al suelo al momento de la siembra; al aporque, a la siembra y aporque, en una dosis de $25 \mathrm{~kg} / \mathrm{ha}$; carbofuran líquido a los bordes de la parcela, a la siembra, a la emergencia y aporque (aplicación calendario), en una dosis de $5 \mathrm{l} / \mathrm{ha}$, barrera natural con Lupinus mutabilis y un testigo absoluto.

\section{Resultados}

\section{Biología}

El gorgojo de los Andes presente en la zona de Lequezana fue identificado como Rhigopsidius tucumanus, de especímenes enviados al Departamento de Nematología-Entomología del Centro Internacional de la Papa en Lima, Perú.

R. tucumanus presentó cuatro estadios larvales, los dos últimos más prolongados y de mayor voracidad (Tabla 1). La cría de larvas, pre-pupas y pupas en tubérculos partidos resultó ser eficiente. El insecto requirió del tubérculo para completar su ciclo de vida, y presentó una generación por año.

Se pudo observar una estrecha relación de la fenología del cultivo con el desarrollo del insecto, que se inició en octubre con la presencia de adultos en la siembra, y de los estadios larvales en el período de tuberización hasta el almacenamiento. 
Tabla 1. Medidas y duración de los estados de desarrollo de Rhigopsidius tucumanus.

\begin{tabular}{|c|c|c|c|c|}
\hline Estado biológico & $\begin{array}{l}\text { Longitud } \\
(\mathrm{mm})\end{array}$ & $\begin{array}{c}\text { Ancho } \\
(\mathrm{mm})\end{array}$ & $\begin{array}{l}\text { Duración } \\
\text { (días) }\end{array}$ & $\begin{array}{c}\text { Casos } \\
\text { observados }\end{array}$ \\
\hline Huevo & $0.8-1.2$ & $0.7-1.0$ & & 72 \\
\hline Incubación & & & $34-45$ & 176 \\
\hline \multicolumn{5}{|l|}{ Larva } \\
\hline 10.Estadio & $1.6-2.3$ & $0.4-0.6$ & $13-13$ & 75 \\
\hline $2^{\circ}$.Estadío & $3.0-5.0$ & $1.0-1.8$ & $9-15$ & 32 \\
\hline $3^{\circ}$.Estadío & $5.8-8.0$ & $1.5-3.0$ & $16-27$ & 47 \\
\hline $4^{\circ}$.Estadío & $10.0-13.0$ & $3.0-4.7$ & $11-27$ & 34 \\
\hline Pre-pupa & & & 27-85 & 28 \\
\hline Pupa & $8.0-11.5$ & $3.0-4.2$ & $36-71$ & 39 \\
\hline \multicolumn{5}{|l|}{ Adulto (Longevidad) } \\
\hline Macho & $6.9-8.9$ & $4.0-4.9$ & 69-202 & 40 \\
\hline Hembra & $8.4-11.2$ & $4.8-1.2$ & $73-190$ & 43 \\
\hline Pre-oviposición & & & $56-76$ & 28 \\
\hline Oviposición & & & $14-78$ & 28 \\
\hline $\begin{array}{l}\mathrm{N}^{\circ} \text { de posturas por } \\
\text { hembra }\end{array}$ & & & $4-16$ & 28 \\
\hline $\begin{array}{l}\mathrm{N}^{\circ} \text { de huevos por } \\
\text { postura }\end{array}$ & & & $1-19$ & 28 \\
\hline $\begin{array}{l}N^{0} \text { de huevos por } \\
\text { hembra }\end{array}$ & & & 18-109 & 28 \\
\hline
\end{tabular}

\section{Daño}

Los muestreos de suelo indicaron la ausencia del insecto en el terreno, antes de la siembra y después de la cosecha.

El índice de daño foliar causado por el adulto de $R$. tucumanus tuvo su inicio con la brotación de plantas, 40 días después de la siembra (dds), y en la época de mayor desarrollo foliar el daño no superó el 2.5\%; luego se redujo paulatinamente con la tuberización, entre 96 y 125 dds (Figura 1). 
Este tipo de daño no afectó el desarrollo del cultivo.

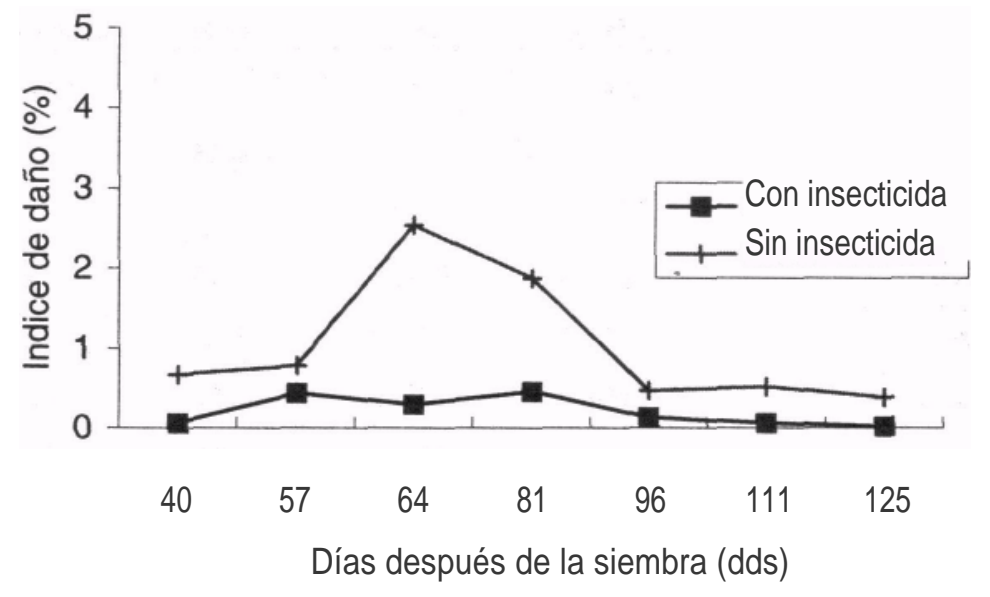

Figura 1. Índice de daño foliar en la variedad Sani Imilla causado por Rhigopsidius tucumanus, con y sin insecticida.

El daño a los tubérculos se inició a la formación de los primeros tubérculos, durante el mes de marzo y se prolongó hasta la cosecha. El porcentaje de daño al tubérculo causado por $R$. tucumanus, sin la aplicación de insecticida, varió entre 28.3 y $85 \%$ y con insecticida no superó el $11 \%$ en las tres localidades (Figura 2).

Al momento de almacenar la papa, el insecto se hallaba en su segundo estadio larval, y dentro del tubérculo completó su desarrollo en el almacén; posteriormente, al momento de la siembra, se encontraba en su estado adulto. La población de la plaga se mantuvo casi constante en el lote de papa almacenada, donde completó su desarrollo; además no se observó daño a los tubérculos contiguos.

Se encontró un reducido número de tubérculos dañados y larvas en las parcelas con insecticida, en cambio éste fue elevado para el tratamiento sin insecticida; el porcentaje de daño promedio en almacén fue de 10 y 82\% respectivamente (Figura 3), similar al obtenido en la cosecha. 


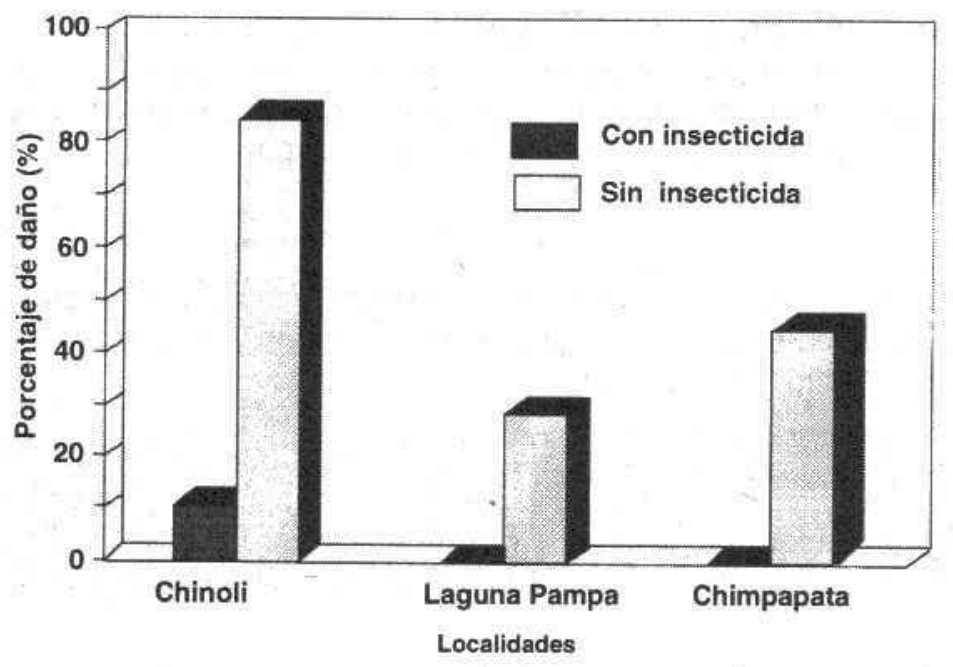

Figura 2. Porcentaje de daño a tubérculos en parcelas del cultivar Sani Imilla, con y sin aplicación de insecticida, en 3 localidades.

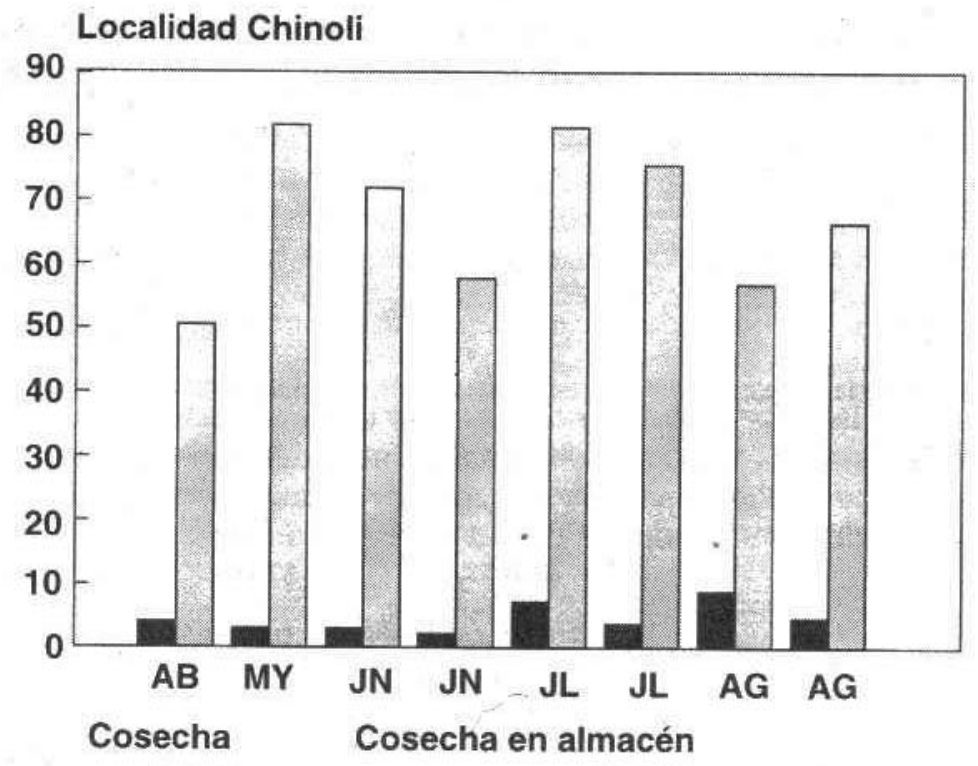

Con insecticida $\square$ Sin insecticida

Figura 3. Número de larvas èn tubérculos almacenados del cultivar Sani Imilla, tratados y no tratados con insecticida. 
En general, durante el almacenamiento se mantuvo constante el número de tubérculos dañados y estados del insecto, y que continuaron desarrollándose dentro de los tubérculos, encontrándose larvas de los cuatro estadios y el estado de prepupa.

\section{Manejo}

En ambas localidades, la barrera vegetal y la aplicación de insecticida al borde de la parcela no tuvieron el efecto deseado y se comportaron igual que el testigo absoluto.

En la localidad de "Yanamok'o" la aplicación de Carbofuran granulado a la siembra; a la siembra y aporque; y líquido a la siembra, emergencia y aporque, tuvieron la misma efectividad (Tabla 2). En la localidad de "Chinoli" los tratamientos se comportaron de manera similar.

Tabla 2. Promedios de rendimiento, porcentaje e índice de daño en la localidad de Yanamok'o.

\begin{tabular}{lccc}
\hline \multicolumn{1}{c}{ Tratamiento } & Porcentaje de daño & Indice de daño & $\begin{array}{c}\text { Rendimiento } \\
\text { (kg/parcela) }\end{array}$ \\
\hline Ins.(G) siembra (S) & $9.66 \mathrm{c}$ & $0.11 \mathrm{~b}$ & $4.15 \mathrm{a}$ \\
Ins.(G) aporque (A) & $57.75 \mathrm{~b}$ & $1.11 \mathrm{a}$ & $2.31 \mathrm{~b}$ \\
Ins.(G) A y S & $4.84 \mathrm{c}$ & $0.06 \mathrm{~b}$ & $4.39 \mathrm{a}$ \\
Ins.(L) calendario & $0.0 \mathrm{c}$ & $0.0 \mathrm{~b}$ & $4.90 \mathrm{a}$ \\
Ins.(L) bordes de la parcela & $64.80 \mathrm{ab}$ & $1.03 \mathrm{a}$ & $2.47 \mathrm{~b}$ \\
Barrera natural & $94.66 \mathrm{a}$ & $1.64 \mathrm{a}$ & $2.20 \mathrm{~b}$ \\
Testigo & $94.87 \mathrm{a}$ & $1.52 \mathrm{a}$ & $1.50 \mathrm{~b}$ \\
CV(\%) & 46.47 & 53.14 & 28.20 \\
DMS & 32.21 & 0.62 & 1.31 \\
\hline
\end{tabular}

\section{Discusión}

Respecto a la biología de $R$. tucumanus, los adultos tuvieron un tamaño máximo similar al reportado por Vilte y Manara (9), pero en la puna Jujeña se pueden encontrar adultos de menor tamaño al registrado en Chinoli. 
Los huevos son esféricos mientras que los de Premnotrypes suturicallus (1), $P$. latithorax (3) y $P$. vorax (4) son de forma capsular, y las larvas son relativamente más grandes y robustas.

R. tucumanus en general empupa y completa su ciclo biológico en el tubérculo, a diferencia de Premnotrypes spp. que empupa en el suelo y forma una cámara pupal (4,1 y 7$)$.

El daño del adulto de $R$. tucumanus al follaje, carece de importancia económica, como ocurre con las otras especies del complejo gorgojo de los Andes (4 y 9). El daño a los tubérculos causado por las larvas posee las mismas características que el ocasionado por Premnotrypes spp. (1, 7 y 5), pudiendo encontrarse de 2 a 9 larvas en un tubérculo.

El comportamiento en almacén de $R$. tucumanus confirmó la importancia de su desarrollo dentro del tubérculo, y que los tubérculos-semillas se constituyen en la principal fuente de diseminación de la plaga, siendo necesaria la selección de tubérculos-semillas libres de la plaga.

El control del gorgojo de los Andes mediante el insecticida Carbofuran es efectivo, aplicado entre la siembra y aporque, que coincide con las revisiones de Yábar (10). Las condiciones agro-ecológicas y el ciclo de vida del insecto, son factores que inciden en la época oportuna de aplicación; así la aplicación solamente en el aporque es menos efectiva que a la siembra. Similares experiencias se tuvieron en Colombia, Perú y Ecuador (5, 8 y 6).

Las barreras naturales y la aplicación de insecticida al borde de la parcela no incidieron en el control del insecto por la forma de infestación en el campo, ya que la plaga se introduce con la semilla, por lo tanto se debe continuar con la búsqueda de otras alternativas de control.

\section{Conclusiones}

- Se identificó a Rhigopsidius tucumanus, como la especie predominante en las pampas de Lequezana de Potosí.

- El insecto requiere del tubérculo para completar su ciclo vital, y tiene un comportamiento sincronizado con relación al desarrollo vegetativo del cultivo. 
- $\quad$ El daño foliar del adulto no tuvo incidencia en el rendimiento.

- $\quad$ El porcentaje de daño en tubérculos que causó $R$. tucumanus varió entre 28.3 y $85 \%$, sin la aplicación de insecticida.

- El control químico con Carbofuran granulado a la siembra; siembra y aporque en una dosis de $25 \mathrm{Kg}$./ha, y Carbofuran líquido en una dosis de $5 \mathrm{l} / \mathrm{ha}$ a la siembra, emergencia y aporque, tuvieron la misma efectividad.

Este trabajo fue ejecutado dentro del marco del convenio del Programa de Investigación de la Papa, PROINPA, que fue establecido entre la Secretaría Nacional de Agricultura y Ganadería de Bolivia, la Cooperación Suiza al Desarrollo (COSUDE) y el Centro Internacional de la Papa (CIP).

\section{Referencias Bibliográficas}

1. Alcalá, C.P.; Alcázar, S.J. 1986. Biología y comportamiento de Premnotrypes suturicallus Kuschel. (Col. Curculionidae). Revista Peruana de Entomología. 19(1):49-52.

2. Barea, M. O. 1991. Diagnóstico de plagas para determinar su importancia. En: Informe Anual 1990-91 PROINPA. Diciembre, 1991. Cochabamba, Bolivia. p. 216-217.

3. Carrasco, F. 1961. Sistemática y biología del gorgojo de los Andes: Premnotrypes latithorax (Pierce,1914). Revista Peruana de Entomología. 4(1):30-42.

4. Calvache, H. 1986. Aspectos biológicos y ecológicos del gusano blanco de la papa: Premnotrypes vorax (Hustache) En: Valencia L. (ed). Control Integrado de Plagas de papa (Memorias). CIP-ICA. Bogotá, Colombia, p. 18-24.

5. Calvache, H. 1989. Importancia de los insectos plagas en el cultivo de la papa. En: PRACIPA. Seminario Taller, sobre Aspectos Entomológicos en el Cultivo de la Papa. Julio 10-13, 1989. Bogotá, Colombia, p.1-17. 
6. Gallegos, P. 1989. Insectos plagas en el cultivo de la papa. En: PRACIPA. Seminario Taller sobre aspectos Entomológicos sobre el Cultivo de la Papa. Julio 10-13,1989. Bogotá, Colombia, p. 31-33.

7. Torrez, W. F. 1989. Principales insectos del cultivo de la papa en Venezuela. En: Seminario Taller sobre Aspectos Entomológicos sobre el Cultivo de la Papa. Julio 10-13, 1989. PRACIPA. Bogotá, Colombia, p. 46-65.

8. Valencia, V.L. 1989. Avances de investigación entomológica en el convenio ICA-CIP. En: Seminario Taller sobre Aspectos Entomológicos sobre el Cultivo de la Papa. Julio 10-13, 1989. PRACIPA. Bogotá, Colombia, p. 66-79.

9. Vilte, H. y Manaro, E.A. 1982. Estudio morfológico y biológico de Rhigopsidius tucumanus Heller (Coleóptera: Curculionidae). Plaga de la papa en la quebrada de Humahuaca y Puna de la Prov. de Jujuy (Argentina). Revista Argentina del Noroeste Argentino. 19(1-4):5-42.

10. Yábar, L. E. 1988. Integración de prácticas culturales para el control del gorgojo de los Andes (Premnotrypes spp.). Revista Latinoamericana de la Papa, ALAP Vol.1 (1). p. 120-129. 